\title{
Review
}

\section{The Vagal Autonomic Pathway of COVID-19 at the Crossroad of Alzheimer's Disease and Aging: A Review of Knowledge}

\author{
Claire-Marie Rangon ${ }^{\mathrm{a}}$, Slavica Krantic ${ }^{\mathrm{b}}$, Emmanuel Moyse $\mathrm{E}^{\mathrm{c}, 1, *}$ and Bertrand Fougère $\mathrm{e}^{\mathrm{d}, \mathrm{e}, 1}$ \\ ${ }^{a}$ Pain and Neuromodulation Unit, Division of Neurosurgery, Hôpital Fondation Ophtalmologique \\ A. De Rothschild, Paris, France \\ ${ }^{\mathrm{b}}$ Sorbonne Université, St. Antoine Research Center (CRSA), Inserm UMRS-938, Hopital St-Antoine, \\ Paris, France \\ ${ }^{\mathrm{c}}$ INRAE Centre Val-de-Loire, Physiology of Reproduction and Behavior Unit (PRC, UMR-85), Team ER2, \\ Nouzilly, France \\ ${ }^{\mathrm{d}}$ Division of Geriatric Medicine, Tours University Hospital, Tours, France \\ ${ }^{\mathrm{e}}$ Education, Ethics, Health (EA 7505), Tours University, Tours, France
}

Accepted 17 November 2020

\begin{abstract}
Coronavirus Disease 2019 (COVID-19) pandemic-triggered mortality is significantly higher in older than in younger populations worldwide. Alzheimer's disease (AD) is related to aging and was recently reported to be among the major risk factors for COVID-19 mortality in older people. The symptomatology of COVID-19 indicates that lethal outcomes of infection rely on neurogenic mechanisms. The present review compiles the available knowledge pointing to the convergence of COVID-19 complications with the mechanisms of autonomic dysfunctions in $\mathrm{AD}$ and aging. The severe acute respiratory syndrome coronavirus-2 (SARS-CoV-2) is prone to neuroinvasion from the lung along the vagus nerve up to the brainstem autonomic nervous centers involved in the coupling of cardiovascular and respiratory rhythms. The brainstem autonomic network allows SARS-CoV-2 to trigger a neurogenic switch to hypertension and hypoventilation, which may act in synergy with aging- and AD-induced dysautonomias, along with an inflammatory "storm". The lethal outcomes of COVID-19, like in $\mathrm{AD}$ and unhealthy aging, likely rely on a critical hypoactivity of the efferent vagus nerve cholinergic pathway, which is involved in lowering cardiovascular pressure and systemic inflammation tone. We further discuss the emerging evidence supporting the use of 1) the non-invasive stimulation of vagus nerve as an additional therapeutic approach for severe COVID-19, and 2) the demonstrated vagal tone index, i.e., heart rate variability, via smartphone-based applications as a non-serological lowcost diagnostic of COVID-19. These two well-known medical approaches are already available and now deserve large-scale testing on human cohorts in the context of both AD and COVID-19.
\end{abstract}

Keyword: Brainstem, dysautonomia, heart rate variability, neuro-immune axis, pulmonary ventilation, vagus nerve, vagus nerve stimulation

COVID-19 is a new infectious disease consisting primarily of acute respiratory distress, caused

\footnotetext{
${ }^{1}$ These authors contributed equally to this work.

*Correspondence to: Dr Emmanuel Moyse, Centre INRAE Val-de-Loire, PRC unit, Team ER2, Route de Monnaie, 37380 Nouzilly, France. Tel.: +33 2474277 00; E-mail: emmanuel. moyse@univ-tours.fr.
}

by a novel coronavirus Severe Acute Respiratory Syndrome CoronaVirus-2 (SARS-CoV-2). COVID19 is characterized by the high mortality rate among the older subjects: up to $22.9 \%$ in people above 90 years in Italy versus less than $2 \%$ in the general population [1]. The elderly population also suffers a higher prevalence of Alzheimer's disease (AD, 32\% 
of people older than 84 years) than in younger subjects [2]. AD was recently demonstrated among major risk factors for COVID-19-related mortality [3, 4]. Furthermore, COVID-19 severity is statistically predicted by the $A P O E \& 4$ genotype, one of the major genetic risk factors for $\mathrm{AD}$ [5]. The recent epidemiological data thus suggest a link between aging and AD regarding fatal outcomes of COVID-19 [6]. Another possible link between aging, AD, and COVID19 concerns a common range of dysfunctions of the autonomic nervous system, or dysautonomia, along with an increased systemic inflammation tone $[7,8]$. The lethal outcomes of COVID-19 might be due to postinfection hypertension and hypoventilation, which is caused by nervous dysfunction of autonomic brainstem centers and reflexes. This present review focuses on the dysfunctions of the vital reflexes mediated by the autonomic system and ensuring the regulation of blood pressure, heart rate, and respiratory ventilation. These neural functions are altogether mediated by the vagus nerve and its brainstem centers, both known as a route for neuroinvasion by coronaviruses and a wellestablished neuroimmune interface [9]. This aspect of SARS-Cov-2-triggered neurogenic pathology has been overviewed in the published surveys of neurological associations of COVID-19 [10-12]. However, most of these previous reviews considered only comorbidities such as meningitis, encephalopathies, Guillain-Barré syndrome of the peripheral nervous system, neuropathies, and increased cerebrovascular risk without considering neurodegenerative diseases, including $\mathrm{AD}$ [10]. Only a recent one has included autonomic dysfunction in an exhaustive description of all published symptoms, including respiratory and circulatory distresses [13]. Based on the growing body of knowledge, this present review expands the recently provided evidence pointing to a pathological link between COVID-19 and AD [13] to underlying neural substrate involving the vagus nerve and its brainstem centers. We further propose to test a recent medical innovation such as non-invasive vagus nerve stimulation via external ear in COVID19, an approach which has recently been proposed to improve cognitive disorders including AD [14]. We finally discuss clinical data supporting the testing of vagal tone index via heart rate variability as an asymptomatic diagnostic marker of infection by SARS-CoV-2.

For the purpose of the present review, our methodology consisted in a bibliographic search crossing the following keywords: COVID-19, Alzheimer's Disease, aging, pathophysiology, SARS-CoV-2, dysau- tonomia, neuroinvasion, Angiotensin-ConvertingEnzyme-2, Angiotensin, vagal afferents, autonomic brainstem centers, lung, dorsal vagal complex, nucleus tractus solitarius, area postrema, cytokine storm, immunological reflex, cholinergic anti-inflammatory pathway, cardiovascular, respiratory drive, vagal nerve stimulation, transauricular vagal nerve stimulation, auricular vagus nerve, heart rate variability.

\section{THE VAGUS NERVE AS A ROUTE OF SARS-COV-2 INVASION OF THE BRAINSTEM: IMPLICATIONS FOR CARDIO-RESPIRATORY FAILURE AND UNCONTROLLED INFLAMMATION}

The vagus nerve is the tenth (Xth) pair cranial nerve, which encompasses mostly viscero-sensory afferents and parasympathetic efferents, to- and from discrete brainstem nuclei. The vagal viscerosensory afferents arise in all visceras as free and ramified nerve terminals belonging to glutamatergic neurons, the cell bodies of which are located in the nodose ganglion (close to the spinal cord in the neck) and project to the nucleus tractus solitarius in the brainstem (Fig. 1) [15, 16]. The vagal efferents consist essentially in the axons of the large cholinergic neurons stacked in the dorsal motor nucleus of the vagus (Fig. 1), that project to all visceras and trigger their actions via cholinergic, local neurons located in intravisceral ganglia [16]. The synaptically interconnected afferents and efferents of the vagus nerve within the dorsal vagal complex of the brainstem yield reflex circuits that provide the anatomical substratum of the vital homeostatic regulations. This connectome is currently being revisited with brain imaging approaches, both in human subjects and in animal models [17].

\section{The pathophysiology of COVID-19}

COVID-19 disease progression comprises three distinct but overlapping phases: an early infection phase, a pulmonary phase, and a severe hyper-inflammation phase [13, 18].

1. In the early infection phase, the virus infiltrates the upper airway tract, lung parenchyma, eyes, and gut. From a recently published extensive survey of literature [13], the first symptoms appear in average 5.2 days after initial contamination and encompass fever, cough, dyspnea, fatigue, nausea, anosmia, and ageusia. This 


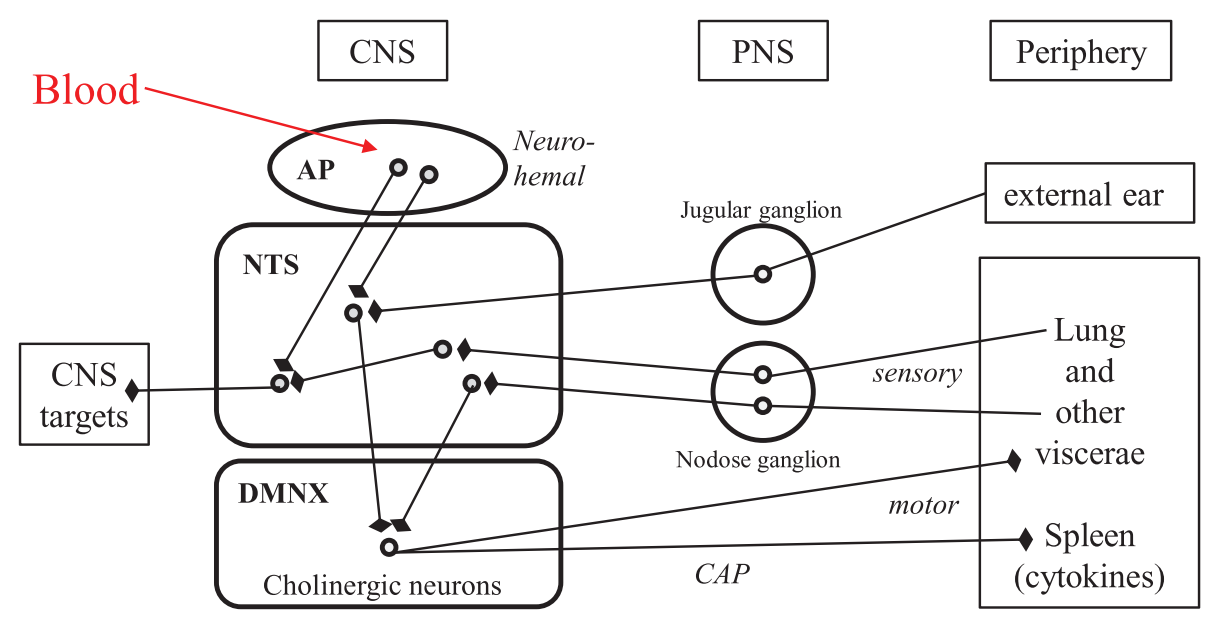

Fig. 1. Neuroanatomical connectivity of the dorsal vagal complex in human brainstem: the "vital node" of pioneer physiologists. Round symbols represent neuronal perikarya. Arrows with blunt end symbolize axonal presynaptic endings. AP, area postrema; CAP, cholinergic anti-inflammatory pathway; CNS, central nervous system; DMNX, dorsal motor nucleus of the vagus; NTS, nucleus tractus solitarius; PNS, peripheral nervous system; IV ${ }^{\text {th }}$, fourth intracerebral ventricle.

initial phase is associated with innate immunity response, which is marked by an increased plasma concentration of C-Reactive Protein (CRP) and activation of monocytes and macrophages, along with a rise of plasmatic amyloid-A protein [13]. Activated monocytes and macrophages have been indeed detected in bronchial and alveolar epithelia by immunohistochemistry after ex-vivo infection of human biopsies with SARS-CoV-2 [19].

2. During the pulmonary phase, collateral tissue injury and subsequent inflammatory processes-vasodilation, increased endothelial permeability, and leukocyte recruitment-lead to further pulmonary damage, hypoxemia (with a frequent atypical clinical presentation called "happy hypoxemia" [20] where severe hypoxemia is associated neither with dyspnea nor abnormal lung compliance), and cardiovascular complications (see below).

3. In a delayed hyperinflammatory phase occurring only in $10-20 \%$ of the patients, mainly older people, the host inflammatory response continues to amplify (even with diminishing viral loads) and yields a systemic inflammation along with cardio-respiratory dysregulation [21], coagulopathy [22], and multiple organ failures. This outcome can lead to death within a period ranging from 6 to 41 days after the onset of COVID-19 symptoms (see above) [13]. This life-challenging phase is likely to have a neurogenic origin, as indicated by case reports of COVID-19 patients who deceased in hospital due to depressed respiratory ventilation after having recovered from pneumonia, and despite ongoing invasive mechanical ventilation [23]. Consistently, experimental infection of mice with a SARS-Cov-2-related virus was shown to trigger early depression of hypercapnic ventilator responses [24]. The symptoms of this late phase altogether indicate a dysfunction of the autonomic nervous system involving an increased sympathetic tone and a decreased parasympathetic tone (Table 1) [13]. The sympathetic/parasympathetic balance at the organism level is controlled by the dorsal vagal complex of the brainstem (see Fig. 1 for details).

This late phase involves a "cytokine storm" (also called macrophage activation syndrome), as observed in various infectious and non-infectious diseases, i.e., an excessive inflammatory response caused by a dysregulated host immune response [25]. The COVID-19 cytokine storm is marked in the systemic circulation by rises of CRP and of activated monocytes-macrophages and affects multiple organs [26]. The mechanism underlying cytokine storm is the failure of inflammatory response regulation back to homeostasis, which normally results from the "inflammatory reflex" defined by Tracey as "a neural loop in which afferent inflammatory signals activate an opposing motor response of the vagus nerve that 
Table 1

Comparative dysautonomia in severe COVID-19, Alzheimer's disease, and nondemented aging

\begin{tabular}{|c|c|c|c|}
\hline Symptoms & Severe COVID-19 & Alzheimer's disease & Non-demented aging \\
\hline & \multicolumn{3}{|c|}{ Arterial blood pressure } \\
\hline \multicolumn{4}{|l|}{ Heart rate } \\
\hline \multicolumn{4}{|l|}{ Cardiac failure risk } \\
\hline \multicolumn{4}{|l|}{ Baroreflex sensitivity } \\
\hline \multicolumn{4}{|l|}{ Respiratory ventilation } \\
\hline \multicolumn{4}{|l|}{ Chemoreflex sensitivity } \\
\hline \multicolumn{4}{|l|}{ Parasympathetic cholinergic tonus } \\
\hline \multicolumn{4}{|l|}{ Sympathetic tonus } \\
\hline \multicolumn{4}{|l|}{ NTS relay } \\
\hline \multicolumn{4}{|l|}{ Heart rate variability } \\
\hline \multicolumn{4}{|l|}{ Systemic inflammation } \\
\hline \multicolumn{4}{|l|}{ Pro-inflammatory cytokine release } \\
\hline \multicolumn{4}{|l|}{ Anti-inflammatory cytokine release } \\
\hline Orthostatic hypotension & - & + & \\
\hline Constipation & - & + & \\
\hline Urinary incontinence & - & + & \\
\hline Dysphagia, swallowing dysfunction & - & + & \\
\hline Impaired pain perception & - & + & \\
\hline
\end{tabular}

Arrows pointing up and down respectively mean increase and decrease. Double arrows indicate major variations. Arrows with dashed line indicate minor variations. (+) and (-) mean presence and absence respectively.

suppresses cytokine production in order to limit or prevent damage" [27].

Beyond the lung, SARS-Cov-2 displays tropism for the vagus nerve and its centers

The initial event of COVID-19 is an infection of human cells by SARS-Cov-2 via stereospecific binding of its envelope $S$ protein (SPIKE) to Angiotensin-Converting Enzyme-2 (ACE2) at the cell membrane and subsequent internalization of the complex [28], which occurs initially in the upper airway and the lung. ACE2 expression has indeed been demonstrated in all cell types of the human airway epithelium by tissue- and single-cell RNA-sequencing from bronchoscopy biopsies of 267 subjects [29]. The viral infection has been observed by applying SARS-CoV-2 particles from nasopharyngeal aspirate of a human COVID-19 patient, on ex-vivo cultures of respiratory tract mucosae and invitro cultures of alveolar epithelial cells from healthy human donors [19]. In human biopsies, SARS-CoV2 has been detected by immunohistochemistry in all differentiated cell types of the airway mucosa and of the alveolar epithelium, aging being related with increased ACE2 protein expression and decreased apoptosis effectors [30]. Its replication period is 2-4 days in both airway- and alveolar tissues [19]. 
The lung-originating infection, propagation, and viral multiplication rate have been confirmed and detailed in a transgenic mouse overexpressing the human ACE2 gene [31]. Importantly, in the human olfactory epithelium, ACE2 was recently revealed by immunohistofluorescence to be localized exclusively in the non-neuronal sustentacular cells, and not in the centrally projecting olfactory sensory neurons [32]. Consistently, single-cell transcriptomal analysis of the murine nasal mucosa lately revealed that SARS-CoV-2 entry genes are expressed exclusively in non-neuronal cells of the olfactory epithelium [33]. These observations render unlikely the nose-tobrain route of SARS-Cov-2 neuroinvasion that has been repetitively postulated since the beginning of COVID-19 pandemy.

ACE2 is ubiquitously expressed in numerous other tissues, encompassing about 150 different cell types [34]. Quantitative comparisons with transcriptomic assays and or comparative immunohistochemistry have all established that the highest ACE2 expressions are found in intestine, followed by testis, gallbladder, kidney, heart muscle, all far above lung and brain levels [34-36]. Remarkably, SARS-CoV2 triggers ACE2 downregulation from the infected cells' plasma membranes and thus lowers tissue capacity to degrade the bioactive vasoconstrictor hormone angiotensin-II into its vasorelaxant and antiinflammatory metabolite angiotensin-(1-7), which in turn yields increased concentration of bioactive angiotensin-II locally in SARS-CoV-2-infected tissues [37].

In the murine brain, extensive mapping of the ACE2 protein by immunohistochemistry showed that ACE2 is expressed exclusively by neurons, and not by glial cells, with the highest densities of ACE2 protein occurring in the three interconnected components of the dorsal vagal complex of the brainstem (Fig. 1): 1) the viscerosensory nucleus tractus solitarius (NTS), 2) the dorsal motor nucleus of the vagus (providing efferent innervation of all viscera, including the heart), and 3) the neuro-hemal structure: area postrema [38-40]. These ACE2-enriched structures of the posterior brain represent the superior autonomic nuclei $[15,41]$, i.e., the centers of the major homeostatic reflexes in mammals (including the baroreflex, the chemoreflex, and the inflammatory reflex, as detailed below). Importantly, the thresholds of these homeostatic reflexes are modulable via cellular plasticity mechanisms such as synaptic efficacies, neurotrophin signaling, axonal sprouting, and neurogenesis [42]. The vagal afferents from the lung arise from peripheral sensory endings that were observed by electron microscopy in direct juxtaposition with the alveolar epithelium in the adult rat, on the basal pole of and in-between the epithelial cells $[43,44]$. These vagal sensory fibers belong to glutamatergic neurons having their perikarya in the peripheral nodose ganglion and projecting directly into the brainstem nucleus tractus solitarius [15]. Since SARS-CoV-2 displays neurotropic property, it could be uptaken by the vagal nerve endings of the lung alveolae up to the NTS [28], and probably spread away trans-synaptically in the nervous system [45]. Consistently, experimental infection of transgenic ACE2-overexpressing mice with another SARS-coronavirus, either by nasal inspiration or by transcranial injection, led to the specific expression of the viral antigen in neurons of the three components of the brainstem dorsal vagal complex by four days after infection [46], i.e., not much longer than the time lag for the initial replication in the lung [19, 31]. This delay for SARS-CoV to migrate from the lung to the brainstem via the vagus nerve to invade the NTS, seems likely to fit with the time-course of atypical hypoxemia and delayed acute respiratory distress syndrome in severe COVID-19 patients, as already suggested by Li et al. [47].

In line with this statement, the neurotropism of SARS-CoV-2 per se has been recently demonstrated in vitro, using cultures of neural cells from human induced pluripotent stem cells [48]. In these neural cells in vitro, moreover, SARS-CoV-2 infection was shown to significantly increase apoptotic death incidence by three times over controls at 1-3 days after infection [48]. The latter observations altogether suggest that the neuroinvasion of the brainstem dorsal vagal complex by SARS-CoV-2 in the course of COVID-19 can rapidly trigger neurodegeneration in this integrative center of the autonomic nervous system; this issue should now be assessed directly by histopathology on postmortem brainstem samples from patients deceased with severe COVID-19.

Besides, SARS-CoV-2 can also gain access to the dorsal vagal complex via blood circulation at the level of the area postrema (Fig. 1), due to the lack of blood-brain-barrier in this neurohemal structure $[15,47]$.

To sum up, the dorsal vagal complex of the brainstem can be a target of SARS-CoV-2 because of its specifically high enrichment in ACE2 and could be reached readily by the virus through two distinct lung-to-brain routes: the vagus nerve and the blood circulation (Fig. 2). 


\section{THE NEUROGENIC DYSAUTONOMIA OF COVID-19: SIMILARITIES WITH AD AND AGING}

In physiological conditions, both cardiorespiratory and immune responses, which are severely impaired in COVID-19, are tightly regulated by the autonomic system centers. Dysregulation of these physiological responses, known as dysautonomia, has been previously reported in $\mathrm{AD}$ as "damage to the autonomic nervous system that impairs function beyond compensatory mechanisms" [49]. In this section, we will first provide general principles behind the physiological regulation of cardiorespiratory and immune functions and then discuss the relevant dysregulations, i.e., dysautonomia in COVID-19, AD, and aging without dementia.

\section{General considerations: the central autonomic network}

Cardio-respiratory regulation is tightly dependent on the baroreflex and the chemoreflex, both involving the vagus nerve and its brainstem centers [50, 51]. The heart rate is tightly linked with the respiratory ventilation cycle since it accelerates during inspiration and decelerates during expiration [52] due to the respiratory sinus arrhythmia, which is itself driven by variations in vagal chronotropic drive [53]. The core breathing rhythm and pattern is determined by a neural pacemaker network in the brainstem: the pre-Bötzinger complex, which is interconnected with cardiovascular regulatory centers of the brainstem via the vagal viscero-sensory NTS [54] (Fig. 1). The steady-state synchrony of the cardio-respiratory rhythm in healthy adults was shown to arise from brainstem centers that altogether determine the vagal efferent activity [55]. The myocardium-innervating vagal efferent fibers arise from two distinct nuclei of the brainstem: the dorsal motor nucleus of the vagus and the nucleus ambiguous [52]. Using pharmacogenetic approaches in awake rats, it has been demonstrated that the neuronal activities of the dorsal motor nucleus of the vagus are required and sufficient to allow exercise-related capacity and endurance adaptation [56].

The dorsal vagal complex is a target of the hypertensive hormone angiotensin-II, which is a key component of systemic cardiovascular regulation [57, 58]. Specific angiotensin-II receptors include two subtypes with opposite physiological effects: AT1-R and AT2-R, which respectively mediate increaseand decrease of arterial pressure and heart rate (reviewed in [37]). Angiotensin-II binds to both AT1 and AT2 receptors, whereas ACE2-generated angiotensin-(1-7) binds only the vasorelaxant AT2$\mathrm{R}$ and Mas receptor (Fig. 2) [37]. The vagal viscerosensory NTS, area postrema, and dorsal motor nucleus of the vagus (DMNV) are all enriched in the hypertensive AT1-R [59]; the hypotensive AT2-R occurs in NTS and DMNV [60] and the hypotensive Mas receptors in NTS only [61]. Angiotensin-II via AT1 receptors of NTS decreases baroreflex sensitivity and increases arterial blood pressure and heart rate [62], whereas experimental overexpression of AT2 receptors in NTS by pharmacogenetics improves baroreflex efficiency in the adult spontaneously hypertensive rat [63].

In addition, the vagus nerve and its brainstem centers down-regulate inflammatory and immune responses via two neural connections: 1) the noradrenergic projection from the vagal sensory nucleus NTS to the neuroendocrine paraventricular nucleus of the hypothalamus, stimulating the secretion of the anti-inflammatory glucocorticoid hormone via the hypothalamic-pituitary-adrenal axis, and 2) the cholinergic projection from the dorsal motor nucleus of the vagus to the spleen and activated immune cells, or cholinergic anti-inflammatory pathway (CAP) [64]. Indeed, in vivo electrical stimulation of the vagus nerve prevents the acute inflammatory response to bacterial toxin injection. Moreover, in vitro, the synthesis of the major pro-inflammatory cytokine Tumor Necrosis Factor-alpha (TNF-alpha) by macrophage cultures is inhibited by the neurotransmitter acetylcholine [65]. Reciprocally, the surgical section of the vagus nerve (or vagotomy) resulted in vivo in enhanced TNF-alpha production and excessive response to endotoxin administration [65]. Furthermore, vagus nerve stimulation reduces inflammation in several experimental models inducing pro-inflammatory cytokines by activating the alpha7-nicotinic acetylcholine receptor of macrophages [66]. In mice deficient for the latter receptor, endotoxin-induced TNF-alpha production is increased, and electrical vagus stimulation fails to reduce serum TNF-alpha levels [66]. More precisely, such macrophage-inhibiting action of the vagus nerve is mediated by acetylcholine-secreting, splenic Tlymphocytes, as demonstrated in a mouse model [67].

The efferent immunosuppressor component of the vagus nerve arises from the DMNV (Fig. 1). It is recruited physiologically in the course of microbial infections or systemic inflammation, by afferent stimulation of vagal sensory fibers that express recep- 


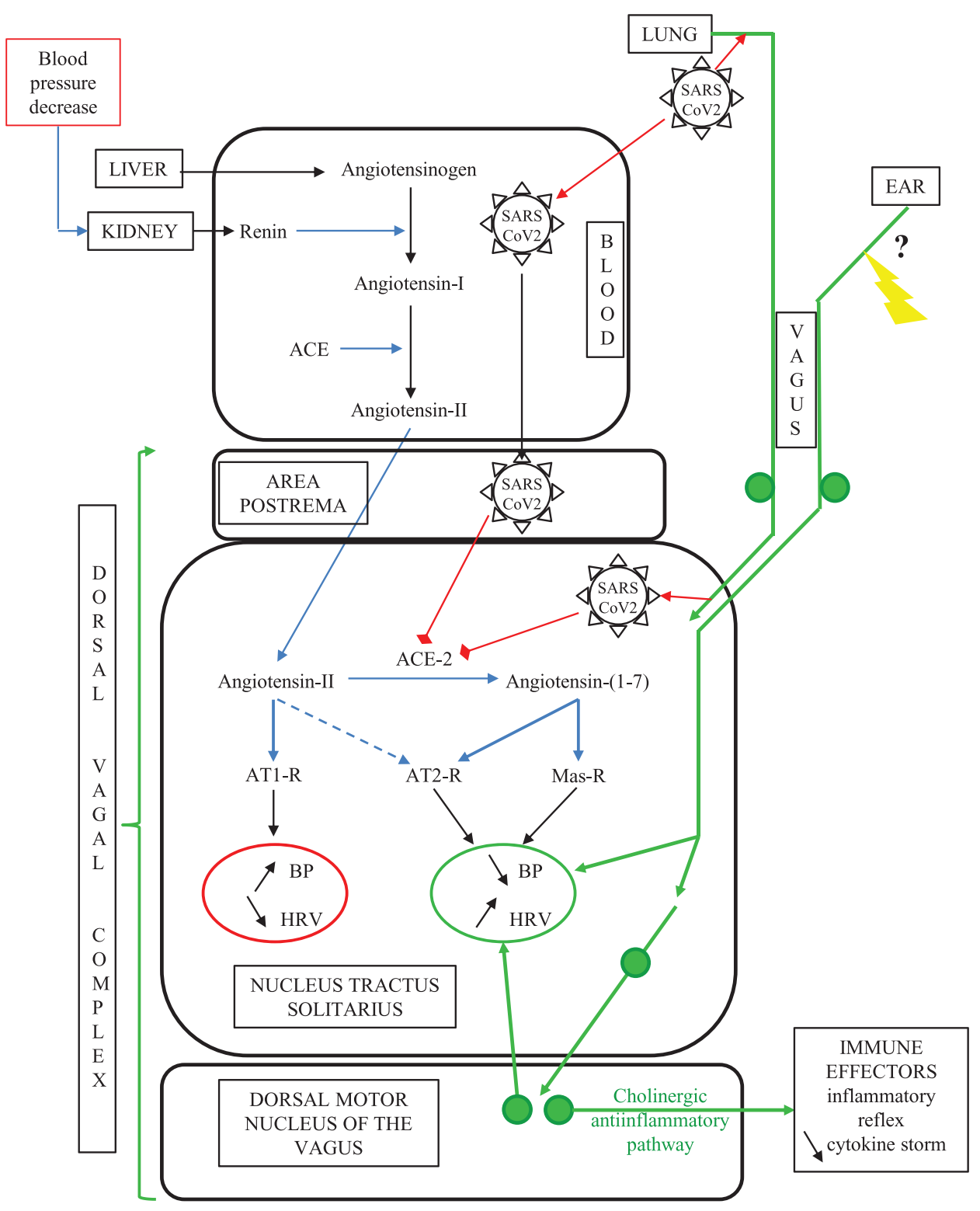

Fig. 2. The vagal routes of SARS-CoV-2 neuroinvasion. In COVID-19, SARS-CoV-2 can gain access to the ACE2-rich dorsal vagal complex either via blood at the level of the neuro-hemal structure area postrema, or via intraneuronal transport through the vagal sensory innervation of the lung alveaolar epithelium. SARS-CoV-2 binding to ACE2 in the dorsal vagal complex yields decreased tissue concentration of ACE2, which in turn increases the ratio between local concentrations of angiotensin-II above its counter-regulatory angiotensin-(1-7) metabolite. This imbalance in the superior autonomic centers worsens the aging-related decline in baroreflex sensitivity and cardiovascular function. Concomitantly, SARS-CoV-2 invasion of the brainstem counteracts the efferent arm of the inflammatory reflex, leading to multi-organ failure through a cytokine storm. Non-medicamentous stimulation of the auricular vagus nerve is likely to restore vagus nerve functions compromised by SARS-CoV2 infection. Green plain circles represent neuronal perikarya; the yellow arrow indicates the therapeutic auricular vagus nerve stimulation (see text).

tors for pro-inflammatory cytokines and project on the viscerosensory NTS (Fig. 1), in the context of the classical "inflammatory reflex" $[64,68]$. In the adult mice, implantation of cuff electrodes at the surface of the cervical vagus nerve and compute- rized neural-to-noise analysis of electrical discharge pattern allowed to identify vagal subsets of sensory fibers [69]. These fibers respond to specific cytokines (especially pro-inflammatory ones) in a dose-dependent manner [69]. It should be stressed 
that the progress in understanding the mechanisms behind neuro-immune communication has allowed for decoding vagal activity patterns in relation to specific diseases [70].

Altogether, the dorsal vagal complex of the brainstem is an integrative center involved in both cardiorespiratory regulation and post-inflammation return to homeostasis.

\section{Dysautonomia in COVID-19}

The first acute consequence of SARS-CoV-2 neuroinvasion into the dorsal vagal complex consists of lowering the tissue concentration of its receptor ACE2 and therefore yields a local increase in angiotensin-II concentration (see above). This hypertensive hormone acts primarily in the dorsal vagal complex to raise blood pressure and heart pumping, which are subsequently potentiated in COVID-19. In parallel, tissue concentration of the ACE2-generated inhibitory feedback metabolite of angiotensin-II: angiotensin-(1-7) decreases [37]. This angiotensinergic imbalance in the superior autonomic center has been established to cause or worsen a decline in baroreflex sensitivity and switch cardiovascular regulation to hypertension [58]. Reciprocally, experimental overexpression of ACE2 by gene transfer into the NTS of an adult spontaneously hypertensive rat was shown to significantly improve the baroreceptor heart rate reflex above controls [71].

The delayed cardiorespiratory symptoms in severe COVID-19 cases include heart palpitations, arrhythmias, myocardial injury, hypertension, chest tightness, and ventilatory dysfunction [72, 73]. Furthermore, in COVID-19 patients, the regulation of respiratory ventilation displays aberrant responses to afferent hypoxia stimuli from the peripheral chemoreceptors (the carotid bodies) [20]. This symptom indicates a drop in the NTS relay of chemoreflex, which may be due to SARS-CoV-2-triggered neurodegeneration (see above section).

A neurogenic model of direct SARS-Cov-2 impact on the brainstem autonomic centers has been recently modelized using a computational approach [74]. Also, most of the clinical characteristics of severe COVID-19, including the "cytokine storm" $[26,75]$ during the third phase of SARS-CoV2 infection, can be explained by dysregulation of the CAP pathway $[76,77]$.

\section{Dysautonomia in Alzheimer's disease}

Interestingly, the recently reported COVID-19related autonomic dysfunction has been character- ized in AD for three decades [7] and proposed to develop in the preclinical phase of the disease [78]. The most common symptoms of AD-associated dysautonomia are cardiac failure or infarction [79], blood pressure dysregulation [80], decreased baroreflex function [81], bronchopneumonia [82], parasympathetic drive hypofunction [83], altered pain perception and reactivity [84], and orthostatic hypotension [7]. In AD patients, lower cognitive performance (especially regarding memory) was related to significantly higher cardiac sympathetic and lower parasympathetic function, independently of age, sex, academic years, diabetes, hypertension, and cholinesterase inhibitor use [85].

These symptoms are attributed to neurodegeneration, including neurofibrillary pathology, that have been mapped in the brainstem centers: in the dorsal motor nucleus of the vagus (which fits with the parasympathetic hypofunction) and to various extents in the NTS, nucleus ambiguous, pre-Bötzinger complex [49, 86-88]. This brainstem neuropathology appears at the Braak's stage VI of $\mathrm{AD}$, i.e., at midcourse of a 20-25 year-long pathological process [88].

\section{Dysautonomia in aging without dementia}

Aging is classically associated with cardiovascular decline and morbidity, which involve decreased parasympathetic activity, blunted baroreflex sensitivity [89], and decline of vagally-modulated heart rate variability [90]. In addition, aging is associated with a systemic rise of inflammation, known as "inflammaging", and is "macrophage-centered" $[8,91]$. Since macrophages are both a major source of systemic inflammation and the main target of the cholinergic anti-inflammatory pathway, inflammaging is likely to result from the hypofunction of the CAP. Strengthening the CAP has indeed been proposed as a new therapeutic perspective in agingrelated disorders [92].

In summary, the dysautonomia is associated with COVID-19, AD, and dementia-free aging, which all share some similarities (Table 1). It is particularly noteworthy that the baroreflex in $\mathrm{AD}$ patients is chronically reduced, which can underlie the much higher incidence of severe COVID-19 in this subpopulation [6]. The failure of the CAP is shared by COVID-19, AD, and dementia-free aging, although along a very different time course (chronic in $\mathrm{AD}$ and aging versus acute in COVID-19), which likely corroborates aging and or $\mathrm{AD}$ as major risk factors for COVID-19-related mortality. The survey of underly- 
ing mechanisms and pathways helps now to propose original, non-serological tools for COVID-19 therapy and or diagnostic.

\section{AURICULAR VAGUS NERVE STIMULATION AS AN INNOVATIVE THERAPEUTIC TOOL IN COVID-19}

Auricular stimulation of the vagus nerve is a noninvasive approach that is increasingly used in therapeutic handling of pharmaco-resistant epilepsy, depression, and other diseases [93]. This therapy relies on the anatomy of the vagus nerve. Indeed, the common cranial trunk of this nerve includes a cervical sensory branch from the ear [94-96].

\section{Vagus nerve stimulation (VNS) as a polyvalent tool in human clinics}

VNS in human patients relies on the permanent implant of a cuff electrode on the left vagus nerve (to avoid cardiac side-effects) at the neck level, which is connected by a subcutaneous wire to a pacemaker-like programmable electronic device. This intervention was approved three decades ago by the Food and Drug Administration (FDA) for treatmentresistant patients with epilepsy and major depressive disorder. The appropriate treatment consists typically in daily 30 -second stimulation at $1 \mathrm{~mA}$ output current with a $20 \mathrm{~Hz}$ frequency of 500 microsecond pulses during several months [97]. The parameters of the vagus nerve stimulator are adjusted during in-office visits by treating physicians to optimize therapeutics by-side effects. VNS also proved beneficial in various cardiovascular and inflammatory diseases [98].

In the $\mathrm{AD}$ field, the above-discussed invasive VNS has already been applied during 6-12 months on cohorts of $\mathrm{AD}$ patients and yielded significant cognitive-enhancing effects $[14,99,100]$.

Studies in animal models demonstrated that VNS resets the baroreflex setpoint and induces inhibition of sympathetic efferents [101, 102], which was also recorded in humans [103]. Of interest, when considering the COVID-19 pandemic, VNS could impact not only on systemic inflammation but also on the coagulant-anticoagulant balance, as suggested by VNS application in endotoxemia rats [104]. In a clinically relevant porcine model of progressive sepsis, VNS significantly attenuated multiple organ dysfunctions (with partial or complete prevention of hyperlactatemia, hyperdynamic circulation, cellular myocardial depression, shift in sympathova- gal balance toward sympathetic dominance, cardiac myocardial dysfunction and reduction of activated monocytes), thus reducing vasopressor and fluid resuscitation requirements [105].

Therefore, it is likely that VNS could attenuate the clinical manifestations of severe COVID-19, as recently suggested [75]. However, VNS requires an invasive surgical procedure for electrode implantation (see above), not an option in COVID-19 pandemics. Conversely, a non-invasive modality of vagus nerve stimulation has been developed, making it more accessible as a possible first-line treatment option (see next section).

\section{Non-invasive vagus nerve stimulation as a novel therapeutic approach for COVID-19?}

Transcutaneous vagus nerve stimulation (tVNS) is a non-invasive procedure consisting of electrical current application through surface electrodes at select locations, most commonly targeting either the cervical branch of the vagus nerve in the neck or the auricular branch of the vagus nerve [106]. Considering these two techniques (cervical or auricular) of tVNS, the cervical location makes selective transcutaneous stimulation of the vagus nerve fibers difficult, because of the presence of both afferent and efferent fibers [107]. By contrast, the auricular branch of the vagus nerve is an exclusively afferent, sensory nerve that innervates part of the skin of the outer ear [94-96]. Hence, trans-auricular vagus nerve stimulation (taVNS) targets only the afferent arm of the vagus nerve.

The optimal location and electrical stimulation parameters providing the greatest tVNS therapeutic effect can be efficiently assessed with heart rate variability (HRV) monitoring. HRV, a biomarker for efferent vagal activation [108], is easier to assess with taVNS than with cervical tVNS [107]. taVNS is mostly used in pharmaco-resistant epilepsy, depression, hypertension, and other diseases [93, 105-107]. A recent study has validated the use of taVNS to inhibit both peripheral and central inflammation and suggested a link between taVNS suppression of inflammation and effectiveness in reducing depression severity [109]. Closer to COVID-19 symptoms, a prospective randomized study on 100 respiratory distressed patients undergoing lobectomy via thoracotomy showed that taVNS could significantly decrease IL-6 and IL-10 levels, reduce the incidence of postoperative pneumonia and shorten duration of hospitalization time [110]. Since drugs commonly 
prescribed to psychiatric patients could protect them from SARS-CoV-2 infection [111], taVNS suppression of neuroinflammation and depression may turn out to be also neuroprotective in the context of COVID-19. So far, however, to the best of our knowledge, only two studies using taVNS (Gammacore ${ }^{\circledR}$ ) in COVID-19 patients $[112,113]$ have been published reporting: 1) a clinical respiratory benefit in two ambulatory COVID-19 patients using cervical tVNS [112]; 2) an ongoing prospective, randomized controlled study on tVNS in patients with moderate to severe COVID-19 respiratory symptoms [113]. Based on this emerging awareness, an International Consortium on Neuromodulation for COVID-19 (ICNC) has been created in early 2020, in order to "provide global leadership for the rapid advancement and clinical adoption of neuromodulation technologies to treat emerging infectious diseases" [114].

Experimental animal studies confirmed the benefits of tVNS by using endotoxemic rodent models and shed light on underlying mechanisms [115, 116]. Remarkably, taVNS also reduced cognitive dysfunction in a postoperative model of aged rats [117] and in a murine model of $\mathrm{AD}$ [118] via a decrease of neuroinflammation. In human clinics, however, taVNS has not been tested so far in $\mathrm{AD}[14,119]$.

It remains now to test taVNS impact on AD symptoms in human cohorts, as already suggested for COVID-19 [75].

\section{AMBULATORY MONITORING OF AURICULAR VAGAL TONE THROUGH HEART RATE VARIABILITY PROVIDES AN ATTRACTIVE PRE-DIAGNOSIS TOOL IN COVID-19 PANDEMIC}

Clinical trials of COVID-19 therapy [77] would undoubtedly benefit from HRV monitoring. HRV is defined as an irregularity of the length of consecutive heart cycles, which reflects the ability of the cardiovascular system to adapt to different situations in everyday life [120]. HRV is calculated based on the R-R interval of the classical PQRS electrocardiogram wave [121]. In the last decades, HRV analysis has emerged as a new useful tool for assessing the changes in autonomic tone that impact on cardiovascular function. HRV is now both considered as an excellent read-out of taVNS [108] and a physiologic marker that provides better and more accurate early warning signs of disease states [122]. Moreover, HRV monitoring based on real-time measurements is easy to get at the bedside.

The most common way of measuring HRV is either time-domain analysis or frequency domain analysis. Parameters calculated from the measurements are related to the functions of both the sympathetic and parasympathetic nervous system [122], which altogether correspond to "autonomic function". The reduction of the fluctuations of the length of consecutive heart cycles has been shown to be associated with pathological conditions, especially in case of inflammation [123, 124].

As a consequence, measurements of changes in autonomic function is crucial in the clinical followup of different diseases as well as of the therapeutic efficacy. Several large epidemiological studies have shown that low HRV is a common risk factor for mortality and morbidity, in particular in Acute Respiratory Distress Syndrome (ARDS) [125] and sepsis [126-128].

In line with pioneer observations of ClaudeBernard, Thayer and Lane have proposed that HRV could also be an index of the level to which the brain generates an adaptive peripheral body's response to environmental changes [129]. A very recent study confirms that HRV analysis provides insight into a "brain-heart-axis" in old patients with mild cognitive impairments, suggesting that this autonomic assessment could help to identify subjects at higher risk of adverse health outcomes, especially among AD patients [130].

Moreover, in addition to the insight in brain-heart axis, HRV appears useful for early screening of infection, with a significant predictive value since HRV alteration is detectable before clinical diagnosis [120] as well as for its very good sensitivity, though low specificity [131, 132]. It is noteworthy that correlations between HRV drop and the onset of sepsis have been found in neonates up to 4 days prior to clinical diagnosis of systemic inflammatory syndrome [133]. Moreover, in the same clinical trial, after treatment and recovery from sepsis, HRV returned to its baseline value [133]. Remarkably, combining HRV monitoring with artificial intelligence-based analysis allows to efficiently predict the onset of sepsis [134]. Since life-threatening forms of COVID-19 involve a systemic inflammatory surge similar to sepsis, future HRV assessment in a large cohort of COVID-19 and healthy subjects is worth doing. Indeed, HRV monitoring is now possible not only in intensive care units [135], but also at a populational level due 
to the wide use of smartphones and both $\mathrm{iOS}$ and Android HRV applications [136, 137]. Notably, a few years ago, a study suggested that such ambulatory monitoring of heart rate in 43 individuals up to 2 years could allow early sepsis detection with $100 \%$ sensitivity [138]. Thus, population-wide longitudinal HRV digital monitoring might enable early screening of SARS-CoV2 infection worldwide.

\section{CONCLUSION}

Altogether, the vagus nerve seems to be "a conduit for neuroinvasion" by SARS-COV-2, "a diagnostic tool and a therapeutic pathway" for COVID-19 (Fig. 2). The essential role of the vagus nerve in the autonomic regulation of vital functions and neuro-immune processes provides a rationale for non-invasive and affordable therapeutic innovations, which should be exploited more broadly by public health policies, for AD as well as for COVID-19 and for pandemics that may arise in future [139]. The available experimental and clinical data point to the need for immediate large-scale testing of a bioelectronic medical strategy for severe COVID19 diagnosis and treatment. In addition, because of the similarities of AD-related dysautonomia with the short term, life-threatening dysautonomia in severe COVID-19, the innovative achievements developed for COVID-19 in this context may turn out to help ameliorate cognitive deficits of $\mathrm{AD}$ patients.

\section{ACKNOWLEDGMENTS}

We express our gratitude to Professor Anne DIDIER (University of Lyon, France) for critical review of the manuscript and communication of late discoveries about SARS-CoV-2 infection of the olfactory mucosa.

\section{CONFLICT OF INTEREST}

The authors have no conflict of interest to report.

\section{REFERENCES}

[1] Onder G, Rezza G, Brusaferro S (2020) Case-fatality rate and characteristics of patients dying in relation to COVID19 in Italy. JAMA 323, 1775-1776.

[2] Alzheimer's Association (2019) 2019 Alzheimer's disease facts and figures. Alzheimers Dement 15, 321-387.

[3] Atkins JL, Masoli JAH, Delgado J, Pilling LC, Kuo CL, Kuchel GA, Melzer D (2020) Preexisting comorbidities predicting COVID-19 and mortality in the UK biobank community cohort. J Gerontol A Biol Sci Med Sci 75, 22242230.

[4] Brown EE, Kular S, Rajji TK, Pollock BG, Mulsant $\mathrm{BH}$ (2020) Anticipating and mitigating the impact of the COVID-19 pandemic on Alzheimer's disease and related dementias. Am J Geriatr Psychiatry 7, 712-721.

[5] Kuo CL, Pilling LC, Atkins JL, Masoli JAH, Delgado J, Kuchel GA, Melzer D (2020) ApoE e4e4 genotype and mortality with COVID-19 in UK Biobank. J Gerontol A Biol Sci Med Sci 75, 1801-1803.

[6] Perry G (2020) Alzheimer's disease patients in the crosshairs of COVID-19. J Alzheimers Dis 76, 1.

[7] Tulba D, Cozma L, Popescu BO, Davidescu EI (2020) Dysautonomia in Alzheimer's disease. Medicina 56, 337.

[8] Fulop T, Larbi A, Dupuis G, Le Page A, Frost EH, Cohen AA, Witkowski JM, Franceschi C (2018) Immunosenescence and inflamm-aging as two sides of the same coin: Friends or foes? Front Immunol 8, 1960.

[9] Porzionato A, Macchi V, Morsut L, Parenti A, De Caro $\mathrm{R}$ (2005) Microvascular patterns in human medullary tegmentum at the level of the area postrema. J Anat 206, 405-410.

[10] Ellul MA, Benjamin L, Singh B, Lant S, Michael BD, Easton A, Kneen R, Defres S, Sejvar J, Solomon T (2020) Neurological associations of COVID-19. Lancet Neurol 19, 767-783.

[11] Wu Y, Xu X, Chen Z, Duan J, Hashimoto K, Yang L, Liu C, Yang C (2020) Nervous system involvement after infection with COVID-19 and other coronaviruses. Brain Behav Immun 87, 18-22.

[12] Fotuhi M, Mian A, Meysami S, Raji CA (2020) Neurobiology of COVID-19. J Alzheimers Dis 76, 3-19.

[13] Yamamoto V, Bolanos JF, Fiallos J, Strand SE, Morris K, Shahrokhinia S, Cushing TR, Hopp L, Tiwari A, Hariri R, Sokolov R, Wheeler C, Kaushik A, Elsayegh A, Eliashiv D, Hedrick R, Jafari B, Johnson JP, Khorsandi M, Gonzalez N, Balakhani G, Lahiri S, Ghavidel K, Amaya M, Kloor H, Hussain N, Huang E, Cormier J, Ashford JW, Wang JC, Yaghobian S, Khorrami P, Shamloo B, Moon C, Shadi P, Kateb B (2020) COVID-19: Review of a $21^{\text {st }}$ century pandemic from etiology to neuro-psychiatric implications. $J$ Alzheimers Dis 77, 459-504.

[14] Broncel A, Bocian R, Klos-Wjtczak P, Kulbat-Warycha K, Konopacki J (2020) Vagal nerve stimulation as a promising tool in the improvement of cognitive disorders. Brain Res Bull 155, 37-47.

[15] Berthoud HR, Neuhuber WL (2000) Functional and chemical anatomy of the afferent vagal system. Auton Neurosci 85, 1-17.

[16] Powley TL, Jaffey DM, McAdams J, Baronowsky EA, Black D, Chesney L, Evans C, Phillips RJ (2019) Vagal innervation of the stomach reassessed: Brain-gut connectome uses smart terminals. Ann NY Acad Sci 145, 14-30.

[17] Hachem LD, Wong SM, Ibrahim GM (2018) The vagus afferent network: Emerging role in translational connectomics. Neurosurg Focus 45(3), E2.

[18] Siddiqi HK, Mehra MR (2020) COVID-19 illness in native and immunosuppressed states: A clinical-therapeutical staging proposal. J Heart Lung Transplant 39, 405-407.

[19] Hui KPY, Cheung MC, Perera RAPM, Ng KC, Bui CHT, Ho JCW, Ng MMT, Kuok DIT, Shih KC, Tsao SW, Poon LLM, Peiris M, Nicholls JM, Chan MCW (2020) Tropism, 
replication competence, and innate immune responses of the coronavirus SARS-CoV-2 in human respiratory tract and conjunctiva: An analysis in ex-vivo and in-vitro cultures. Lancet Respir Med 8, 687-695.

[20] Ur A, Verma K (2020) Happy Hypoxemia in COVID-19 A neural hypothesis. ACS Chem Neurosci 11, 1865-1867.

[21] Wu Z, McGoogan JM (2020) Characteristics of and important lessons from the coronavirus disease 2019 (COVID-19): Outbreak in China: Summary of a report of 72314 cases from the Chinese center for disease control and prevention. JAMA 323, 1239-1242.

[22] Connors JM, Levy JH (2020) COVID-19 and its implications for thrombosis and anticoagulation. Blood 135, 2033-2040.

[23] Manganelli F, Vargas M, Iovino A, Iacovazzo C, Santoro L, Servillo G (2020) Brainstem involvement and respiratory failure in COVID-19. Neurol Sci 41, 1663-1665.

[24] Zhuang J, Zang N, Ye C, Xu F (2019) Lethal avian influenza A $(\mathrm{H} 5 \mathrm{~N} 1)$ virus replicates in pontomedullary chemosensitive neurons and depresses hypercapnic ventilatory response in mice. Am J Physiol Lung 316, 1525-1536.

[25] Tisoncik JR, Korth MJ, Simmons CP, Farrar J, Martin TR, Katze MG (2012) Into the eye of the cytokine storm. Microbiol Mol Biol Rev 76, 16-32.

[26] Ur A, Verma K (2020) Cytokine storm in COVID19: A neural hypothesis. ACS Chem Neurosci 11, 1868-1870.

[27] Tracey KJ (2002) The inflammatory reflex. Nature 420, 853-859.

[28] Hoffmann M, Kleine-Weber H, Schroeder S, Kruger N, Herrler T, Erichsen S, Schiergens TS, Herrler G, Wu NH, Nitsche A, Müller MA, Drosten C, Pöhlmann S (2020) SARS-CoV-2 cell entry depends on ACE2 and TMPRSS2 and is blocked by a clinically proven protease inhibitor. Cell 181, 271-280.e8.

[29] Zhang H, Rostami MR, Leopold PL, Mezey JG, O'Beirne SL, Strulovici-Barel Y, Crystal RG (2020) Expression of the SARS-CoV-2 ACE2 receptor in the human airway epithelium. Am J Respir Crit Care Med 202, 219-229.

[30] Inde Z, Yapp C, Joshi GN, Spetz J, Fraser C, Deskin B, Ghelfi E, Sodhi C, Hackam D, Kobzik L, Croker B, Brownfield D, Jia H, Sarosiek KA (2020) Agedependent regulation of SARS-CoV-2 cell entry genes and cell death programs correlates with COVID-19 disease severity. bioRxiv 2020.09.13.276923; doi: https://doi. org/10.1101/2020.09.13.276923.

[31] Jiang RD, Liu MQ, Chen Y, Shan C, Zhou YW, Shen XR, Li Q, Zhang L, Zhu Y, Si HR, Wang Q, Min J, Wang X, Li B, Zhang HJ, Baric RS, Zhou P, Yang XL, Shi ZL (2020) Pathogenesis of SARS-CoV-2 in transgenic mice expressing human angiotensin-converting enzyme 2 . Cell 182, 50-58.

[32] Chen M, Shen W, Rowan NR, Kulaga H, Hillel A, Ramanathan M Jr, Lane AP (2020) Elevated ACE-2 expression in the olfactory neuroepithelium: Implications for anosmia and upper respiratory SARS-CoV-2 entry and replication. Eur Respir J 56, 2001948.

[33] Brann DH, Tsukahara T, Weinreb C, Lipovsek M, Ven den Berge K, Gong B, Chance R, Macaulay IC, Chou HJ, Fletcher RB, Das D, Street K, Roux de Bezieux H, Choi YG, Risso D, Dudoit S, Purdom E, Mill J, Hachem RA, Matsunami H, Logan DW, Goldstein BJ, Grubb MS, Ngai J, Datta SR (2020) Non-neuronal expression of SARS-CoV-2 entry genes in the olfactory system suggests mechanisms underlying COVID-19-associated anosmia. Sci Adv 6, eabc5801.

[34] Hikmet F, Méar L, Edvinsson A, Micke P, Uhlén M, Lindskog C (2020) The protein expression profile of ACE2 in human tissues. Mol Syst Biol 16, e9610.

[35] Wang Y, Wang Y, Luo W, Huang L, Xiao J, Li F, Qin S, Song X, Wu Y, Zeng Q, Jin F, Wang Y (2020) A comprehensive investigation of the mRNA and protein level of ACE2, the putative receptor of SARS-CoV-2, in human tissues and blood cells. Int J Med Sci 17, 15221531.

[36] Li MY, Li L, Zhang Y, Wang XS (2020) Expression of the SARS-CoV-2 cell receptor gene ACE2 in a wide variety of human tissues. Infect Dis Pov 9, 45.

[37] Verdecchia P, Cavallini C, Spanevello A, Angeli F (2020) The pivotal link between ACE2 deficiency and SARSCoV-2 infection. Eur J Int Med 76, 14-20.

[38] South AM, Diz DI, Chappell MC (2020) COVID-19, ACE2, and the cardio-vascular consequences. Am J Physiol Heart Circ Physiol 318, H1084-H1900.

[39] Lin Z, Chen Y, Zhang W, Chen AF, Lin S, Morris M (2008) RNA interference shows interactions between mouse brainstem angiotensin AT1 receptors and angiotensinconverting enzyme 2. Exp Physiol 93, 676-684.

[40] Mendelsohn FA, Allen AM, Chai SY, McKinley MJ, Oldfield BJ, Paxinos G (1990) The brain angiotensin system: Insights from mapping its components. Trends Endocrinol Metab 1, 189-198.

[41] Gourine AV, Machhada A, Trapp S, Spyer KM (2016) Cardiac vagal preganglionic neurons: An update. Auton Neurosci 199, 24-28.

[42] Moyse E, Bauer S, Charrier C, Coronas V, Krantic S, Jean A (2006) Neurogenesis and neural stem cells in the dorsal vagal complex of adult rat brain: New vistas about autonomic regulations - a review. Auton Neurosci 126-127, 50-58.

[43] Doobay MF, Talman LS, Obr TD, Tuan X, Davisson RL, Lazartigues E (2007) Differential expression of neuronal ACE2 in transgenic mice with overexpression of the brain renin-angiotensin system. Am J Physiol Regul Integr Comp Physiol 292, R373-R381.

[44] Adriaensen D, Timmermans JP, Brouns I, Berthoud HR, Neuhuber WL, Scheuermann DW (1998) Pulmonary intraepithelial vagal nodose afferent nerve terminals are confined to neuroepithelial bodies: An anterograde tracing and confocal microscopy study in adult rats. Cell Tissue Res 293, 395-405.

[45] Chen SH, Haam J, Walker M, Scappini E, Naughton J, Martin NP (2019) Overview: Recombinant viral vectors as neuroscience tools. Curr Protoc Neurosci 87, e67.

[46] Netland J, Meyerholz DK, Moore S, Cassell M, Perlman S (2008) Severe acute respiratory syndrome coronavirus infection causes neuronal death in the absence of encephalitis in mice transgenic for human ACE2. $J$ Virol 82, 7264-7275.

[47] Li YC, Bai WZ, Hashikawa T (2020) The neuroinvasive potential of SARS-CoV2 may play a role in the respiratory failure of COVID-19 patients. J Med Virol 92, 552-555.

[48] Jacob F, Pather SR, Huang WK, Zhang F, Wong SZH, Zhou H, Cubitt B, Fan W, Chen CZ, Xu M, Pradhan M, Zhang DY, Zheng W, Bang AG, Song H, de A Torre JC, Ming GL (2020) Human pluripotent stem cell-derived neural cells and brain organoids reveal SARS-CoV-2 neu- 
rotropism predominates in choroid plexus epithelium. Cell Stem Cell 27, 937-950.e9.

[49] Engelhardt E, Laks J (2008) Alzheimer disease neuropathology: Understanding autonomic dysfunction. Dement Neuropsychol 2, 183-191.

[50] Chigr F, Merzouki M, Najimi M (2020) Autonomic brain centers and pathophysiology of COVID-19. ACS Chem Neurosci 11, 1520-1522.

[51] Tassorelli C, Mojoli F, Baldanti F, Bruno R, Benazzo M (2020) COVID-19: What if the brain had a role in causing the deaths? Eur J Neurol 27, e41-e42.

[52] Gourine AV, Ackland GL (2019) Cardiac vagus and exercise. Physiology 34, 71-80.

[53] Yasuma F, Hayano J (2004) Respiratory sinus arrhythmia: Why does the heartbeat synchronize with respiratory rhythm? Chest 125, 683-690.

[54] Ghali MGZ (2019) Respiratory rhythm generation and pattern formation: Oscillators and network mechanisms. $J$ Integr Neurosci 18, 481-517.

[55] Farmer DGS, Dutschmann M, Paton JFR, Pickering AE, McAllen RM (2016) Brainstem sources of cardiac vagal tone and respiratory sinus arrhythmia. J Physiol 594, 72497265.

[56] Machhada A, Trapp S, Marina N, Stephens RCM, Whittle J, Lythgoe MF, Kasparov S, Ackland GL, Gourine AV (2017) Vagal determinants of exercise capacity. Nat Commun 18, 15097.

[57] Allen AM, McKinley MJ, Oldfield BJ, Dampney RA, Mendelsohn FA (1988) Angiotensin II binding and the baroreflex pathway. Clin Exp Hyperten A 10(Suppl 1), 63-78.

[58] Arnold AC, Gallagher PE, Diz DI (2013) Brain reninangiotensin system in the nexus of hypertension and aging. Hypertens Res 36, 5-13.

[59] Lenkei Z, Palkovits M, Corvol P, Llorens-Cortes C (1998) Distribution of angiotensin type-1 receptor messenger RNA expression in the adult rat brain. Neuroscience 82, 827-841.

[60] De Kloet AD, Wang L, Ludin JA, Smith JA, Pioquinto DJ, Hiller H, Steckelings UM, Scheuer DA, Sumners C, Krause EG (2016) Reporter mouse strain provides a novel look at angiotensin type- 2 receptor distribution in the central nervous system. Brain Struct Funct 221, 891-912.

[61] Becker LK, Etelvino GM, Walther T, Santos RAS, Campagnole-Santos MJ (2007) Immunofluorescence localization of the receptor Mas in cardiovascular-related areas of the rat brain. Am J Physiol Heart Circ Physiol 293, H141-H124.

[62] Paton JF, Kasparov S (1999) Differential effects of angiotensin II on cardiorespiratory reflexes mediated by nucleus tractus solitarii - a microinjection study in the rat. J Physiol 521, 213-225.

[63] Ruchaya PJ, Speretta GF, Blanch GT, Li H, Sumners C, Menani JV, Colombari E, Colombari DSA (2016) Overexpression of AT2R in the solitary-vagal complex improves baroreflex in the spontaneously hypertensive rat. $\mathrm{Neu}$ ropeptides $\mathbf{6 0}, 29-36$.

[64] Tracey KJ (2007) Physiology and immunology of the cholinergic anti-inflammatory pathway. J Clin Invest 117, 289-296.

[65] Borovikova LV, Ivanova S, Zhang M, Yang H, Botchkina GI, Watkins LR, Wang H, Abumrad N, Eaton JW, Tracey KJ (2000) Vagus nerve stimulation attenuates the systemic inflammatory response to endotoxin. Nature $\mathbf{4 0 5}$, 458-462.
[66] Wang H, Yu M, Ochani M, Amella CA, Tanovic M, Susarla $\mathrm{S}$, Li JH, Wang H, Yang H, Ulloa L, Al-Abed Y, Czura CJ, Tracey KJ (2003) Nicotinic acetylcholine receptor $\alpha 7$ subunit is an essential regulator of inflammation. Nature 421, 384-388.

[67] Rosas-Ballinas M, Olofsson PS, Ochani M, Valdes-Ferrer SI, Levine YA, Reardon C, Tusche MW, Pavlov VA, Andersson U, Chavan S, Mak TW, Tracey KJ (2011) Acetylcholine-synthetizing $\mathrm{T}$ cells relay neural signals in a vagus nerve circuit. Science 334, 98-101.

[68] Ulloa L (2005) The vagus nerve and the nicotinic antiinflammatory pathway. Nat Rev Drug Discov 4, 673-684.

[69] Zanos TP, Silverman HA, Levy T, Tsaava T, Battinelli E, Lorraine PW, Ashe JM, Chavan SS, Tracey KJ, Bouton CE (2018) Identification of cytokine-specific sensory neural signals by decoding murine vagus nerve activity. Proc Natl Acad Sci U S A 115, E4843-E4852.

[70] Zanos TP (2019) Recording and decoding of vagal neural signals related to changes in physiological parameters and biomarkers of disease. Cold Spring Harb Perspect Med $\mathbf{9}$, a034157.

[71] Yamazato M, Ferreira AJ, Yamazato Y, Diez-Freire C, Yuan L, Gillies R, Raizada MK (2011) Gene transfer of angiotensin-converting enzyme 2 in the nucleus tractus solitarius improves baroreceptor heart rate reflex in spontaneously hypertensive rats. $J$ Renin Angiotensin Aldosterone Syst 12, 456-461.

[72] Zheng YY, Ma YT, Zhang JY, Xie X (2020) COVID19 and the cardiovascular system. Nat Rev Cardiol 17, 259-260.

[73] Kang Y, Chen T, Mui D, Ferrari V, Jagasia D, ScherrerCosbie M, Chen Y, Han Y (2020) Cardiovascular manifestations and treatment considerations in covid-19. Heart 106, 1132-1141.

[74] Baig AM (2020) Computing the effects of SARS-CoV-2 on respiration regulatory mechanisms in COVID-19. ACS Chem Neurosci 11, 2416-2421.

[75] Fudim M, Qadri YJ, Ghadimi K, MacLeod DB, Molinger J, Piccini JP, Whittle J, Wischmeyer PE, Patel MR, Ulloa L (2020) Implications for neuromodulation therapy to control inflammation and related organ dysfunction in COVID-19. J Cardiovasc Transl Res 26, 1-6.

[76] Farsalinos K, Niaura R, Le Houezec J, Barbouni A, Tsatsakis A, Kouretas D, Vantarakis A, Poulas K (2020) Nicotine and SARS-CoV-2: COVID-19 may be a disease of the nicotinic cholinergic system. Toxicol Rep 7, 658-663.

[77] Bonaz B, Sinniger V, Pellissier S (2020) Targeting the cholinergic anti-inflammatory pathway with vagus nerve stimulation in patients with Covid-19? Bioelectron Med $\mathbf{6}$, 15.

[78] Femminella GD, Rengo G, Komici K, Iacotucci P, Petraglia L, Pagano G, de Lucia C, Canonico V, Bonaduce D, Leosco D, Ferrara N (2014) Autonomic dysfunction in Alzheimer's Disease: Tools for assessment and review of the literature. J Alzheimers Dis 42, 369-377.

[79] Santos CY, Machan JT, Wu WC, Snyder PJ (2017) Autonomic cardiac function in preclinical Alzheimer's disease. J Alzheimers Dis 59, 1057-1065.

[80] Lattanzi S, Luzzi S, Provinciali L, Silvestrini M (2014) Blood pressure variability predicts cognitive decline in Alzheimer's Disease patients. Neurobiol Aging 35, 22822287.

[81] Meel van den Abeelen ASS, Lagro J, Gommer ED, Reulen JPH, Claassen JAHR (2013) Baroreflex function is 
reduced in Alzheimer's Disease: A candidate biomarker? Neurobiol Aging 4, 1170-1176.

[82] Manabe T, Mizukami K, Akatsu H, Hashizume Y, Ohkubo T, Kudo K, Hizawa N (2017) Factors associated with pneumonia-caused death in older adults with autopsyconfirmed dementia. Intern Med 56, 907-914.

[83] Collins O, Dillon S, Finucane C, Lawlor B, Kenny RA (2012) Parasympathetic autonomic dysfunction is common in mild cognitive impairment. Neurobiol Aging 33, 2324-2333.

[84] Beach PA, Huck JT, Zhu DC, Bozoki AC (2017) Altered behavioral and autonomic pain responses in Alzheimer's disease are associated with dysfunctional affective, selfreflective and salience network resting connectivity. Front Aging Neurosci 9, 297.

[85] Nonogaki Z, Umegaki H, Makino T, Suzuki Y, Kuzuya M (2017) Relationship between cardiac autonomic function and cognitive function in Alzheimer's disease. Geriatr Gerontol Int 1, 92-98.

[86] Braak E, Alafuzoff I, Arzberger T, Kretzschmar H, Del Tredici K (2006) Staging of Alzheimer diseaseassociated neurofibrillary pathology using paraffin sections and immunocytochemistry. Acta Neuropathol 112, 389-404.

[87] Parvizi J, Van Hoesen GW, Damasio A (2001) The selective vulnerability of brainstem nuclei to Alzheimer's disease. Ann Neurol 49, 53-66.

[88] Attems J, Thomas A, Jellinger K (2012) Correlations between cortical and subcortical tau pathology. $\mathrm{Neu}$ ropathol Appl Neurobiol 38, 582-590.

[89] Kaye DM, Esler MD (2008) Autonomic control of the aging heart. Neuromolecular Med 10, 179-186.

[90] De Meersman RE, Stein PK (2007) Vagal modulation and aging. Biol Psychol 74, 165-173.

[91] Franceschi C, Bonafé M, Valensin S, Olivieri F, De Luca M, Ottaviani E, Benedictis G (2000) Inflamm-aging. An evolutionary perspective in immunosenescence. Ann NY Acad Sci 908, 244-254.

[92] Benfante R, Di Lascio S, Cardani S, Fornasari D (2019) Acetylcholinesterase inhibitors targeting the cholinergic anti-inflammatory pathway: A new therapeutic perspective in aging-related disorders. Aging Clin Exp Res, doi: 10.1007/s40520-019-01359-4.

[93] Mercante B, Deriu F, Rangon CM (2018) Auricular neuromodulation: The emerging concept beyond the stimulation of vagus and trigeminal nerves. Medicines (Basel) 5,10 .

[94] Murray AR, Atkinson L, Mahadi MK, Deuchars SA, Deuchars J (2016) The strange case of the ear and the heart: The auricular vagus nerve and its influence on cardiac control. Auton Neurosci 199, 48-53.

[95] Peuker ET, Filler TJ (2002) The nerve supply of the human auricle. Clin Anat 15, 35-37.

[96] Butt MF, Albusoda A, Farmer AD, Aziz Q (2020) The anatomical basis for transcutaneous auricular vagus nerve stimulation. J Anat 236, 588-611.

[97] Koopman FA, Chavan SS, Miljko S, Grazio S, Sokolovic S, Schuurman PR, Mehta AD, Levine YA, Faltys M, Zitnik R, Tracey KJ, Tak PP (2016) Vagus nerve stimulation inhibits cytokine production and attenuates disease severity in rheumatoid arthritis. Proc Natl Acad Sci U S A 113, 8284-8289.

[98] Johnson RL, Wilson CG (2018) A review of vagus nerve stimulation as a therapeutic intervention. J Inflamm Res 11, 203-213.
[99] Sjogren MJ, Hellstrom PT, Jonsson MA, Runnerstam M, Silander HC, Ben Menachem E (2002) Cognitionenhancing effect of vagus nerve stimulation in patients with Alzheimer's Disease: A pilot study. J Clin Psychiatry 63, 972-980.

[100] Merrill CA, Jonsson MA, Minthon L, Ejnell H, C-son Silander H, Blennow K, Karlsson M, Nordlund A, Rolstad S, Warkentin S, Ben-Menachem E, Sjögren MJ (2006) Vagus nerve stimulation in patients with Alzheimer's disease: Additional follow-up results of a pilot study through one year. J Clin Psychiatry 67, 1171-1178.

[101] Saku K, Kishi T, Sakamoto K, Hosokawa K, Sakamoto T, Murayama Y, Kakino T, Ikeda M, Ide T, Sunagawa K (2014) Afferent vagal nerve stimulation resets baroreflex neural arc and inhibits sympathetic nerve activity. Physiol Rep 2, e12136.

[102] Kawada T, Li M, Zheng C, Shimizu S, Uemura K, Turner MJ, Yamamoto H, Sugimachi M (1985) Chronic vagal nerve stimulation improves baroreflex neural arc function in heart failure rats. J Appl Physiol 116, 1308-1314.

[103] Clancy JA, Mary DA, Witte KK, Greenwood JP, Deuchars SA, Deuchars J (2014) Non-invasive vagus nerve stimulation in healthy humans reduces sympathetic nerve activity. Brain Stimul 7, 871-877.

[104] Van Westerloo DJ, Giebelen IA, Meijers JC, Daalhuisen J, de Vos AF, Levi M, van der Poll T (2006) Vagus nerve stimulation inhibits activation of coagulation and fibrinolysis during endotoxemia in rats. J Thromb Haemost 4, 1997-2002.

[105] Kohoutova M, Horak J, Jarkovska D, Martinkova V, Tegl V, Nalos L, Vistejnova L, Benes J, Sviglerova J, Kuncova J, Matejovic M, Stengl M (2019) Vagus nerve stimulation attenuates multiple organ dysfunction in resuscitated porcine progressive sepsis. Crit Care Med 47, e461-e469.

[106] Yap JYY, Keatch C, Lambert E, Woods W, Stoddart PR, Kameneva T (2020) Critical review of transcutaneous vagus nerve stimulation: Challenges for translation to clinical practice. Front Neurosci 14, 284.

[107] Yuan H, Silberstein SD (2016) Vagus nerve and vagus nerve stimulation, a comprehensive review: Part II. Headache 56, 259-266.

[108] Burger AM, D’ Agostini M, Verkuil B, Van Diest I (2020) Moving beyond belief: A narrative review of potential biomarkers for transcutaneous vagus nerve stimulation. Psychophysiology 57, e13571.

[109] Liu CH, Yang MH, Zhang GZ, Wang XX, Li B, Li M, Woelfer M, Walter M, Wang L (2020) Neural networks and the anti-inflammatory effect of transcutaneous auricular vagus nerve stimulation in depression. $J$ Neuroinflammation 17, 54.

[110] Salama M, Akan A, Mueller MR (2020) Transcutaneous stimulation of auricular branch of the vagus nerve attenuates the acute inflammatory response after lung lobectomy. World J Surg 44, 3167-3174.

[111] Villoutreix BO, Beaune PH, Tamouza R, Krishnamoorthy R, Leboyer M (2020) Prevention of COVID-19 by drug repurposing: Rationale from drugs prescribed for mental disorders. Drug Discov Today 25, 1287-1290.

[112] Staats P, Giannakopoulos G, Blake J, Liebler E, Levy RM (2020) The use of non-invasive vagus nerve stimulation to treat respiratory symptoms associated with COVID19: A theoretical hypothesis and early clinical experience. Neuromodulation 23, 784-788.

[113] Tornero C, Vallejo R, Cedeño D, Orduna J, Pastor E, Belaouchi M, Escamilla B, Laredo M, Garzando 
MDM (2020) A prospective, randomized, controlled study assessing vagus nerve stimulation using the gammaCore ${ }^{\circledR}-$ Sapphire device for patients with moderate to severe CoViD-19 respiratory symptoms (SAVIOR): A structured summary of a study protocol for a randomised controlled trial". Trials 21, 576.

[114] International Consortium on Neuromodulation for COVID-19. Neuromodulation has the capacity to address acute and chronic effects of COVID-19. http:// www.covidneuromod.org, Accessed 25 July 2020.

[115] Zhao YX, He W, Jing XH, Liu JL, Rong PJ, Ben H, Liu K, Zhu B (2012) Transcutaneous auricular vagus nerve stimulation protects endotoxemic rat from lipopolysaccharide-induced inflammation. Evid Based Complement Alternat Med 2012, 627023.

[116] Hong GS, Zillekens A, Schneiker B, Pantelis D, de Jonge WJ, Schaefer N, Kalff JC, Wehner S (2019) Non-invasive transcutaneous auricular vagus nerve stimulation prevents postoperative ileus and endotoxemia in mice. Neurogastroenterol Motil 31, e13501.

[117] Cai L, Lu K, Chen X, Huang JY, Zhang BP, Zhang H (2019) Auricular vagus nerve stimulation protects against postoperative cognitive dysfunction by attenuating neuroinflammation and neurodegeneration in aged rats. Neurosci Lett 703, 104-110.

[118] Kaczmarczyk R, Tejera D, Simon BJ, Heneka MT (2018) Microglia modulation through external vagus nerve stimulation in a murine model of Alzheimer's disease. $J$ Neurochem 146, 76-85.

[119] Chang CH, Lane HY, Lin CH (2018) Brain stimulation in Alzheimer's disease. Front Psychiatry 9, 201.

[120] Ahmad S, Tejuja A, Newman KD, Zarychanski R, Seely AJE (2009) Clinical review: A review and analysis of heart rate variability and the diagnosis and prognosis of infection. Crit Care 13, 232.

[121] Shaffer F, Ginsberg JP (2017) An overview of heart rate variability metrics and norms. Front Public Health 5, 258.

[122] Griffin MP (2005) Heart rate characteristics: Novel physiomarkers to predict neonatal infection and death. Pediatrics 116, 1070-1074.

[123] Sloan RP, McCreath H, Tracey KJ, Sidney S, Liu K, Seeman T (2007) RR interval variability is inversely related to inflammatory markers: The CARDIA study. $\mathrm{Mol} \mathrm{Med}$ 13, 178-184.

[124] Williams DP, Koenig J, Carnaveli L, Sgoifo A, Jarczok MN, Sternberg EM, Thayer JF (2019) Heart rate variability and inflammation: A meta-analysis of human studies. Brain Behav Immun 80, 219-226.

[125] Chen IC, Kor CT, Lin CH, Kuo J, Tsai JZ, Ko WJ, Kuo CD (2018) High-frequency power of heart rate variability can predict the outcome of thoracic surgical patients with acute respiratory distress syndrome on admission to the intensive care unit: A prospective, single-centric, case-controlled study. BMC Anesthesiol 18, 34 .

[126] Barnaby DP, Fernando SM, Herry CL, Scales NB, Gallagher EJ, Seely AJE (2019) Heart rate variability, clinical and laboratory measures to predict future deterioration in patients presenting with sepsis. Shock 51, 416-422.
[127] de Castilho FM, Ribeiro ALP, da Silva JLP, Nobre V, de Sousa MR (2017) Heart rate variability as predictor of mortality in sepsis: A prospective cohort study. PLoS One 12, e 0180060

[128] de Castilho FM, Ribeiro ALP, Nobre V, Barros G, de Sousa MR (2018) Heart rate variability as predictor of mortality in sepsis: A systematic review. PLoS One 13, e0203487.

[129] Thayer JF, Lane RD (2009) Claude Bernard and the heart-brain connection: Further elaboration of a model of neurovisceral integration. Neurosci Biobehav Rev 33, 81-88.

[130] Nicolini P, Mari D, Abbate C, Inglese S, Bertagnoli L, Tomasini E, Rossi PD, Lombardi F (2020) Autonomic function in amnestic and non-amnestic mild cognitive impairment: Spectral heart rate variability analysis provides evidence for a brain-heart axis. Sci Rep 10, 11661.

[131] Hsu CT, Tai HCH, Chung JY, Chen JH, Chen WL (2020) Depressed sympathovagal modulation indicates sepsis in patients with suspected infection. Medicine (Baltimore) 99, e18961.

[132] Chiew CJ, Liu N, Tagami T, Wong TH, Koh ZX, Ong MEH (2019) Heart rate variability based machine learning models for risk prediction of suspected sepsis patients in the emergency department. Medicine (Baltimore) 98, e14197.

[133] Kovatchev BP, Farhy LS, Cao H, Griffin MP, Lake DE, Moorman JR (2003) Sample asymmetry analysis of heart rate characteristics with application to neonatal sepsis and systemic inflammatory response syndrome. Pediatr Res 54, 892-898.

[134] Kamaleswaran R, Akbilgic O, Hallman MA, West AN, Davis RL, Shah SH (2018) Applying artificial intelligence to identify physiomarkers predicting severe sepsis in the PICU. Pediatr Crit Care Med 19, e495-e503.

[135] Johnston BW, Barrett-Jolley R, Krige A, Welters ID (2020) Heart rate variability: Measurement and emerging use in critical care medicine. J Intensive Care Soc 21, 148-157.

[136] Liu I, Ni S, Peng K (2020) Enhancing the robustness of smartphone photoplethysmography: A signal quality index approach. Sensors (Basel) 20, 1923.

[137] MacKinnon GE, Brittain EL (2020) Mobile health technologies in cardiopulmonary disease. Chest 157, 654-664.

[138] Li X, Dunn J, Salins D, Zhou G, Zhou W, SchüsslerFiorenza-Rose SM, Perelman D, Colbert E, Runge R, Rego S, Sonecha R, Datta S, McLaughlin T, Snyder MP (2017) Digital health: Tracking physiomes and activity using wearable biosensors reveals useful health-related information. PLoS Biol 15, e2001402.

[139] Serrano-Castro PJ, Estivill-Torrús G, Cabezudo-García P, Reyes-Bueno JA, Petersen NC, Aguilar-Castillo MJ, Suarez-Perez J, Jimenez-Hernandez MD, Moya-Molina MA, Oliver-Martos B, Arrabal-Gomez C, de Fonsecca FR (2020) Impact of SARS-CoV-2 infection on neurodegenerative and neuropsychiatric diseases: A delayed pandemic? Neurologia 35, 245-251. 